\title{
RASTREIO DA PRÉ-ECLÂMPSIA UTILIZANDO AS CARACTERÍSTICAS MATERNAS E A PRESSÃO ARTERIAL MÉDIA DE GESTANTES
}

\author{
SCREENING FOR PRE-ECLAMPSIA USING MATERNAL CHARACTERISTICS AND \\ MEAN ARTERIAL PRESSURE OF PREGNANT WOMEN
}

\section{Bárbara Gomes Santos Silva ${ }^{1} *$ Karla Polyana de Moura Guimarães Souza ${ }^{2} *$ Maria Luziene de Sousa Gomes ${ }^{3} *$ Ivana Rios Rodrigues ${ }^{4} *$ João Joadson Duarte Teixeira ${ }^{5}$ Nadya dos Santos Moura $^{6}$ * Mônica Oliveira Batista Oriá ${ }^{7}$}

\begin{abstract}
RESUMO
Objetivo: Rastrear os fatores de risco e a pressão arterial média de gestantes quanto ao desenvolvimento da pré-eclâmpsia. Método: Estudo transversal, desenvolvido em cinco unidades de saúde da família no período de março a junho de 2019. Os dados foram obtidos por meio de instrumento contendo variáveis sociodemográficas e clínicas, analisados mediante estatística descritiva e inferencial com aplicação do teste Qui-quadrado. Resultados: Participaram do estudo 60 gestantes. Dentre os fatores de risco, houve associação entre o fator de risco pré-eclâmpsia prévia $(\mathrm{p}=0,0001)$ e a Pressão Arterial Média. Dentre os antecedentes clínicos das participantes, infecção urinária foi a mais prevalente das condições $16(26,7 \%)$, seguida da hipertensão arterial 7 $(11,7 \%)$ e infertilidade $4(6,7 \%)$. Conclusão: Por meio do rastreio foi evidenciada a associação entre os fatores de risco e a pressão arterial média das gestantes.
\end{abstract}

Palavras-chave: Fatores de risco; Pré-eclâmpsia; Cuidado pré-natal; Atenção primária à saúde; Enfermagem.

\begin{abstract}
Objective: Track risk factors and mean arterial pressure of pregnant women regarding the development of pre-eclampsia. Method: Cross-sectional study, carried out in five family health units from March to June 2019. Data were obtained using an instrument containing sociodemographic and clinical variables, analyzed using descriptive and inferential statistics with the application of the Chi-square test. Results: 60 pregnant women participated in the study. Among the risk factors, there was an association between the risk factor for previous pre-eclampsia $(\mathrm{p}=0.0001)$ and mean arterial pressure. Among the clinical history of the participants, urinary tract infection was the most prevalent of the conditions $16(26.7 \%)$, followed by arterial hypertension 7 $(11.7 \%)$ and infertility $4(6.7 \%)$. Conclusion: Through screening the association between risk factors and the mean arterial pressure of pregnant women was evidenced.
\end{abstract}

Keyword: Risk factors; Pre-eclampsia; Prenatal care; Primary health care, Nursing.

\footnotetext{
1 Enfermeira, mestranda no Programa de Pós-graduação em Enfermagem, Universidade Federal do Ceará - UFC. E-mail: barbaragmss@gmail.com, ID Orcid: https://orcid.org/0000-0001-8892-2293

${ }^{2}$ Enfermeira, Universidade Federal do Piauí - UFPI. Email: polyanagui@hotmail.com, ID Orcid: https://orcid.org/0000-0001-96915712

${ }^{3}$ Enfermeira, doutoranda no Programa de Pós-graduação em Enfermagem, Universidade Federal do Ceará - UFC. E-mail: luziene94@ hotmail.com, ID Orcid: https://orcid.org/0000-0001-8583-0959

${ }^{4}$ Enfermeira, doutoranda no Programa de Pós-graduação em Enfermagem, Universidade Federal do Ceará - UFC. E-mail: ivana_rius@ @otmail.com, ID Orcid: https://orcid.org/0000-0002-6558-6407

5 Enfermeiro, doutorando no Programa de Pós-graduação em Enfermagem, Universidade Federal do Ceará - UFC. E-mail: joadson2606@gmail.com, ID Orcid: https://orcid.org/0000-0002-0438-5433

6 Enfermeira, Professora Assistente, Universidade Federal do Piauí. E-mail: nadyasantosm@yahoo.com.br, ID Orcid: https://orcid.org/0000-0001-5081-5206

${ }^{7}$ Enfermeira, Professora Associada II. Programa de Pós-graduação em Enfermagem, Universidade Federal do Ceará - UFC. E-mail: profmonicaoria@gmail.com, ID Orcid: https://orcid.org/0000-0002-1483-6656
} 


\section{INTRODUÇÃO}

No cenário mundial, as Síndromes Hipertensivas da Gestação (SHG) são consideradas as principais causas de morte materna e neonatal. Nos países desenvolvidos, esta prevalência varia entre 2 e $8 \%$ das gestações, enquanto no Brasil pode alcançar valores acima de $10 \%$, constituindo a primeira causa de morte materna, apresentando elevada taxa de morbimortalidade perinatal ${ }^{(1-2)}$. Os gastos do sistema de saúde público brasileiro ultrapassam 14 milhões de dólares por ano em custos diretos para o tratamento de Préeclâmpsia (PE) e eclâmpsia, uma despesa considerável para um país em desenvolvimento ${ }^{(1)}$.

$$
\text { É imprescindível a identificação }
$$
precoce do risco de PE a fim de permitir a adoção de medidas profiláticas e a individualização da vigilância obstétrica ${ }^{(3)}$. Entre os preditores mais estudados para o rastreio da PE estão as Características Maternas (CM) e a Pressão Arterial Média (PAM) por oferecerem bom custo-benefício, serem baratos e de fácil aplicação $^{(4)}$. A predição de $\mathrm{PE}$ consiste na tentativa de identificar gestantes de alto risco para modificar os cuidados pré-natais e instituir medidas de prevenção e tratamento em tempo oportuno, a fim de reduzir complicações e mortes em decorrência da doença ${ }^{(5)}$.

O American College of Obstetricians an obtenção de uma detalhada história médica das gestantes a fim de avaliar os fatores de risco, constituindo excelente método para o rastreio de PE. Considera-se que, caracterizar o perfil das gestantes e todas as informações sobre sua saúde, é de extrema relevância, pois contribui para identificação de riscos e com isso elaboração de estratégias preventivas a fim de reduzir os agravos, possibilitando a oferta de uma assistência com qualidade ${ }^{(6)}$. Adicionalmente, outro método de rastreio de excelente desempenho para predição de PE é a PAM, obtida a partir da tomada da média de duas medições em ambos os braços, simultaneamente, por aparelhos automatizados, calibrados e validados ${ }^{(7-8)}$.

Dessa forma, o estudo justifica-se pelo interesse em verificar as $\mathrm{CM}$ e a PAM em gestantes atendidas nas Unidades de Saúde da Família (USF), buscando identificar fatores que possam predispor ao surgimento da PE, pois, torna-se fundamental investigar precocemente fatores que contribuam para o adoecimento materno, a fim de oportunizar em tempo hábil estratégias para prevenção, diagnóstico precoce, tratamento eficaz e promover melhor qualidade de vida materna.

Assim, este estudo teve como objetivo rastrear os fatores de risco e a pressão arterial média de gestantes quanto ao desenvolvimento da pré-eclâmpsia.

\section{d Gynecologists (ACOG) recomenda a MÉTODOS}


Estudo transversal realizado em cinco

USF com maior número de gestantes cadastradas em consulta de pré-natal em um município do interior do Piauí, no período de março a junho de 2019. A partir de uma lista com o nome de todas as gestantes cadastradas e ativas nas consultas de pré-natal, foram aplicados os seguintes critérios de seleção: gestante até a $12^{\mathrm{a}}$ semana gestacional (recomendação aponta que o melhor período para rastreamento da PE é no primeiro acompanhada pelas equipes das unidades de saúde selecionadas para o estudo. Para evitar viés de interpretação foram excluídas as que se encontravam com PE na gestação atual. A população de gestantes cadastradas nas consultas de pré-natal nas USF foi de 292 mulheres, no entanto, o fato de iniciar o rastreio da $\mathrm{PE}$ ainda no primeiro trimestre gestacional ocasionou a redução da amostra para 92 partipantes, destas , 32 se recusaram a colaborar. Totalizando a amostra final de 60 gestantes.

Em seguida foram agendadas em dias e horários específicos a aplicação do instrumento, buscando contemplar a participação do maior número de gestantes, explicando-se individualmente os objetivos do estudo e aplicando o instrumento em sala reservada da unidade básica de saúde.

Para coleta de dados foi utilizado um formulário estruturado elaborado pelos autores incluindo: variáveis sociodemográficas (idade, escolaridade, trimestre gestacional) $^{(7)}$ e estar sendo

ocupação, renda familiar e cor) e características maternas (número de gestações, número e tipo de parto: cesáreo ou normal, número de abortos, gravidez planejada: sim ou não, atividade física: sim ou não), antecedentes clínicos (hipertensão arterial crônica, infecção urinária e infertilidade), fatores de risco para desenvolvimento da PE (primiparidade, pré-eclâmpsia prévia, hipertensão arterial, gestação múltipla, história familiar de préeclâmpsia, obesidade e idade materna avançada) ${ }^{(8)}$ e os valores dos resultados da PAM.

Para aferição da PAM teve-se como instrumento, dois monitores de pressão arterial de braço automáticos da marca Omron $\mathrm{Hem}^{\circledR}$, referência 7113. Este aparelho já foi validado e é largamente utilizado em estudos com gestantes ${ }^{(9)}$. O preparo das usuárias estava em conformidade com as diretrizes da Sociedade Brasileira de Cardiologia $^{(10)}$. A partir dos valores obtidos como pressão arterial sistólica e diastólica, foram calculados os valores da PAM, com a fórmula: [(sistólica $+2 \mathrm{x}$ diastólica): 3]. Adotou-se como ponto de corte o valor de 90 mmHg para a PAM, esse valor obteve taxa de detecção de $62 \%$ para PA em estudo anterior ${ }^{(11)}$.

Os dados coletados foram codificados para elaboração de um dicionário de dados. Depois, transcritos, com o processo de dupla digitação em planilhas do aplicativo Microsoft Excel e corrigidos os erros para serem https://doi.org/10.31011/reaid-2021-v.95-n.34-art.1069 Rev Enferm Atual In Derme v. 95, n. 34, 2021 e-021083 
exportados

para

O

programa Statistical Package for the Social

Science for Windows Versão 20.0 (SPSS

Versão 20.0). O tratamento dos dados foi

possível com testes de estatística descritiva e

inferencial, por meio do teste do qui-quadrado

de Pearson, considerando o nível de

significância $\mathrm{p}<0,005$, cujos dados estão

representados por tabelas.

Os pesquisadores respeitaram os

princípios éticos contidos na Resolução $\mathrm{N}^{\circ}$ 466/12 que rege pesquisas envolvendo seres humanos. Foram solicitadas as assinaturas dos
Termos de Consentimento Livre e Esclarecido (TCLE) e Termo de Assentimento Livre e Esclarecido (TALE) para as menores de 18 anos de idade. O estudo foi aprovado pelo Comitê de Ética em Pesquisa da Universidade Federal do Piaú, sob Parecer $n^{\circ}$ 2.838.357/2018.

\section{RESULTADOS}

As características sociodemográficas estão descritas na Tabela 1 e o perfil clínico e obstétrico está na Tabela 2.

Tabela 1 - Características sociodemográficas das gestantes (n=60) Picos-PI, Brasil, 2019.

\begin{tabular}{lcc}
\hline Variáveis & $\mathbf{n}$ & $\mathbf{\%}$ \\
\hline $\begin{array}{l}\text { Idade (anos) } \\
<40\end{array}$ & 58 & 96,7 \\
$>40$ & 02 & 3,3 \\
Escolaridade (anos de estudo) & & \\
$5-8$ & 10 & 16,7 \\
& 50 & 83,3
\end{tabular}

\section{Ocupação}

Dona de casa $21 \quad 35,0$

Trabalho formal $24 \quad 40,0$

Trabalho informal $06 \quad 10,0$

Estudante

$09 \quad 15,0$

Renda Familiar (em salários mínimos)

$<1$

$1-3$

$>3$

Cor

Branca

Negra

Parda
$17 \quad 28,3$

$37 \quad 61,7$

$06 \quad 10,0$ 
Acerca dos antecedentes clínicos, a de hipertensão arterial $(n=7$;

infecção urinária foi a condição mais e infertilidade $(n=4 ; 6,7 \%)$ prevalente $(n=16 ; 26,7 \%), \quad$ seguida

Tabela 2 - Descrição do perfil clínico e obstétrico das gestantes (n=60) Picos-PI, Brasil, 2019.

\begin{tabular}{|c|c|c|}
\hline Variáveis & $\mathbf{n}$ & $\%$ \\
\hline \multicolumn{3}{|l|}{ Gestações } \\
\hline 1 & 26 & 43,3 \\
\hline $2-3$ & 31 & 51,6 \\
\hline 4 & 03 & 5 \\
\hline \multicolumn{3}{|l|}{ Parto } \\
\hline Nenhum & 31 & 51,7 \\
\hline 1 & 21 & 35 \\
\hline$>2$ & 08 & 13,4 \\
\hline \multicolumn{3}{|l|}{ Aborto } \\
\hline 0 & 47 & 78,3 \\
\hline 1 & 10 & 16,7 \\
\hline 2 & 03 & 5,0 \\
\hline \multicolumn{3}{|l|}{ Gravidez Planejada } \\
\hline $\operatorname{Sim}$ & 28 & 46,7 \\
\hline Não & 32 & 53,3 \\
\hline \multicolumn{3}{|c|}{ Atividade física antes da gestação } \\
\hline Sim & 33 & 55,0 \\
\hline Não & 27 & 45,0 \\
\hline \multicolumn{3}{|c|}{ Atividade física durante a gestação } \\
\hline Sim & 10 & 16,7 \\
\hline Não & 50 & 83,3 \\
\hline \multicolumn{3}{|l|}{ Antecedentes clínicos } \\
\hline Hipertensão Arterial Crônica & 07 & 11,7 \\
\hline Infecção Urinária & 16 & 26,7 \\
\hline Infertilidade & 04 & 6,7 \\
\hline
\end{tabular}

Todas as gravidezes tiveram concepção espontânea. Entre as mulheres que já haviam parido, a maioria foi submetida à cesariana $(\mathrm{n}=15 ; 53,3 \%)$; afirmaram ter feito uso de tabaco e outras drogas antes e durante a gestação $(\mathrm{n}=1 ; 1,7 \%)$ e bebida alcoólica $(\mathrm{n}=5 ; 8,3 \%)$. 
A Tabela 3 traz os resultados encontrados mediante a associação entre a PAM e os fatores de risco para PE. Houve associação entre $\mathrm{o}$ fator de risco préeclâmpsia prévia e a PAM ( $\mathrm{p}=0,0001)$.

Tabela 3-Associação entre a PAM e os fatores de risco para PE na gestação atual. Picos-PI, Brasil, 2019.

\begin{tabular}{|c|c|c|c|}
\hline \multirow{2}{*}{$\begin{array}{l}\text { Variáveis } \\
\text { Fator de risco para pré-eclâmpsia }\end{array}$} & \multicolumn{2}{|c|}{ PAM } & \multirow[b]{2}{*}{$\mathbf{p}^{*}$} \\
\hline & $<90 \mathrm{mmHg}$ & $>90 \mathrm{mmHg}$ & \\
\hline \multicolumn{4}{|l|}{ Primiparidade } \\
\hline Sim & 0 & 31 & 0,297 \\
\hline Não & 1 & 28 & \\
\hline \multicolumn{4}{|l|}{ Pré-eclâmpsia prévia } \\
\hline $\begin{array}{l}\text { Sim } \\
\text { Não } \\
\text { Hipertensão Arterial Crônica }\end{array}$ & $\begin{array}{l}2 \\
0\end{array}$ & $\begin{array}{c}0 \\
58\end{array}$ & $<0,0001$ \\
\hline Sim & 0 & 03 & 0,817 \\
\hline Não & 01 & 56 & \\
\hline \multicolumn{4}{|l|}{ Gestação Múltipla } \\
\hline Sim & 0 & 01 & 0,896 \\
\hline Não & 01 & 58 & \\
\hline \multicolumn{4}{|l|}{ História Familiar de Pré-eclâmpsia } \\
\hline Sim & 0 & 11 & 0,633 \\
\hline Não & 01 & 48 & \\
\hline \multicolumn{4}{|l|}{ Obesidade } \\
\hline Sim & 0 & 10 & 0,652 \\
\hline Não & 01 & 49 & \\
\hline \multicolumn{4}{|l|}{ Idade Materna Avançada } \\
\hline Sim & 0 & 02 & 0,851 \\
\hline Não & 01 & 57 & \\
\hline
\end{tabular}

p = significância estatística

DISCUSSÃO

As limitações do estudo estão relacionadas ao delineamento transversal do método utilizado uma vez que impossibilitou o acompanhamento e o desfecho das gestações, assim como o tamanho da amostra, que impediu a generalização dos resultados. Porém, vale ressaltar a modesta quantidade de estudos relacionados à predição 
de PE no Brasil, bem como, dificuldades encontradas durante a coleta de dados, tais como: recusa por parte do público, principalmente relacionada à falta de tempo disponível para responder o instrumento de coleta de dados e participar da avaliação clínica, por se tratar de mulheres que dividiam-se entre ocupações com trabalho, atividades domésticas e familiares.

Esta pesquisa viabilizou dados recentes ao meio científico sobre as CM e PAM das gestantes, bem como sua relação com o desenvolvimento de fatores de risco para PE, propiciando assim, a ampliação das informações ao meio acadêmico, aos enfermeiros e demais profissionais que prestam assistência a esse público, podendo sensibilizá-los para o incremento na assistência materna. Essas informações também podem estender-se às gestantes, por permitir expor conhecimentos sobre a PE e a importância da identificação precoce da doença.

Estudos relacionados à $\mathrm{PE}$ sugerem a prevalência de mulheres jovens. Em contrapartida, a ACOG declara que a idade materna avançada (>40 anos) configura-se como fator de risco para o desenvolvimento de $\mathrm{PE}^{(8)}$, o que afirmação exclui a maioria das participantes desse estudo do grupo de risco. Contudo, o fator idade isolado não deve ser generalizado, devendo o enfermeiro, durante a consulta de pré-natal, atentar-se aos demais fatores de riscos para o desenvolvimento da
PE e realizar o devido encaminhamento à atenção especializada, quando necessário.

Quanto à renda das gestantes, ressaltase que o baixo nível socioeconômico pode contribuir para o desenvolvimento da PE e está associado a piores desfechos gestacionais $^{(12)}$. Os dados referentes a renda familiar aqui apresentados vão ao encontro dos dados de uma investigação realizada em uma capital do Nordeste, ao constatar que mais da metade da amostra possuia renda de um a três salários mínimos ${ }^{(13)}$. Em geral, gestantes de baixo nível socioeconômico têm piores condições de saúde e acesso aos serviços.

A etnia/raça/cor da pele é uma variável difícil de avaliar na população brasileira uma vez que nossa população é muito miscigenada. Isso se confirma inclusive quando a maioria das gestantes afirma ser parda (58,3\%). A literatura referente a $\mathrm{PE}$ constata que mulheres negras apresentam maior predisposição ao desenvolvimento de hipertensão arterial crônica, o que aumenta a incidência de PE sobreposta à hipertensão ${ }^{(14)}$. Todavia, é importante ressaltar que apesar da PE apresentar maior predisposição em mulheres de pele negra, essa síndrome ainda manifesta-se com frequência em mulheres de outras cores ou raças ${ }^{(15)}$.

A prática de atividade física durante o período gestacional é um tema que vem ganhando espaço nas discussões no meio científico. A maioria das gestantes relatou praticar exercício físico antes da https://doi.org/10.31011/reaid-2021-v.95-n.34-art.1069 Rev Enferm Atual In Derme v. 95, n. 34, 2021 e-021083 
gestação e não durante. Uma revisão sistemática traz evidências dos benefícios da prática de atividade física na gestação, representando fator protetor ao desenvolvimento da $\mathrm{SHG}^{(16)}$. Mesmo nos estudos em que não foram encontrados benefícios da atividade física durante a gestação, não foram observados prejuízos desta prática à saúde materno-fetal, exceto às gestantes que já possuiam a patologia instalada $^{(16)}$.

Dentre os antecedentes clínicos das participantes, infecção urinária foi a mais relevante das condições, posto que a maioria já apresentou a doença. Este achado também foi percebido em outro estudo relacionado e $\mathrm{PE}^{(13)}$. Episódios de infecção do trato urinário durante a gestação pode ser preocupante pois está relacionada à rotura de membranas ovulares, trabalho de parto prematuro, recémnascidos com baixo peso, sepse materna e infecção neonatal $^{(17)}$.

Embora não se tenha o desfecho obstétrico da amostra envolvida, a maioria $(53,3 \%)$ foi submetida à cesarianas anteriores, o que também foi encontrado em outro estudo $^{(18)}$. Apesar dos altos índices de cesarianas no país, sabe-se que seja qual for a forma clínica da SHG, a via de parto recomendada pelas diretrizes nacionais e internacionais é a vaginal ${ }^{(18)}$. Um estudo realizado com gestantes de alto risco portadoras de síndrome hipertensiva verificou que o parto via cesárea foi considerado um fator de risco desfavorável ao desfecho https://doi.org/10.31011/reaid-2021-v.95-n.34-art.1069 Rev Enferm Atual In Derme v. 95, n. 34, 2021 e-021083

perinatal $^{(19)}$, entretanto no agravamento da hipertensão, intervenções imediatas devem ser realizadas, incluindo o parto cesáreo, que pode também ter complicações do próprio procedimento, tais como reações ao anestésicos, sangramentos e infecções, riscos esses menos comuns ao parto normal ${ }^{(19)}$.

$\mathrm{Na}$ associação entre os Fatores de Risco para PE e a PAM, a variável Pré-eclâmpsia prévia foi a que apresentou associação $(\mathrm{p}=0,0001)$. Essa relação também foi evidenciada em outros estudo que identificou esta associação em $6,2 \%$ dos $\operatorname{casos}^{(8)}$. É importante ressaltar que a PE anterior é um importante fator de risco para nova gestação com PE, investigação apontou um aumento de duas vezes no risco cardiovascular e morte em mulheres com pré-eclâmpsia anterior $^{(20)}$. Aumentos de risco específicos são estimados em 3,7 vezes para hipertensão e 1,8 vezes para acidente vascular cerebral $^{(20)}$. Ressalta-se a importância da assistência integral às gestantes do pré-natal de baixo risco, pois esse público pode estar suscetível a complicações que muitas vezes se manifestam de forma silenciosa como as SHG.

Os dados apresentados colaboram com o conhecimento acerca das características sociodemográficas e perfil clínico e obstétrico, assim como da PAM das gestantes atendidas no âmbito da Atenção Primária em Saúde (APS), permitindo que sejam comparados com estudos nacionais e internacionais. O fato de algumas variáveis 
não atingirem significância estatística pode ser explicado pelo número reduzido da amostra e por serem gestantes assistidas no pré-natal de baixo risco. Logo, sugere-se novas pesquisas na área, incluindo uma amostra maior e que envolva pacientes do pré-natal de alto e baixo risco visando a exposição de distintos perfis obstétricos.

No que cabe a atuação do enfermeiro, ressalta-se, ainda, a importância da consulta de pré-natal de qualidade, analisando o histórico familiar e pessoal da gestante, a fim de prevenir que estes se integrem como manifestadores de evoluções desfavoráveis durante o período gestacional.

\section{CONCLUSÃO}

Através do rastreio das características maternas e da pressão arteriam média das gestantes foi evidenciada a associação entre o fator de risco Pré-eclâmpsia prévia e a PAM. Logo, torna-se oportuno identificar os fatores de risco para o desenvolvimento da $\mathrm{PE}$ durante o pré-natal de baixo risco. A caracterização do perfil social, clínico e obstétrico, bem como a medida da PAM são considerados excelentes estratégias de baixo custo a serem implementadas na saúde pública para rastreio da PE. Vale ressaltar, que mesmo tratando-se de uma amostra pequena e advinda do pré-natal de baixo, os preditores de PE mostraram-se eficazes para o rastreio dessa síndrome.

\section{REFERÊNCIAS}

1. Henderson JT, Thompson JH, Burda BU, Cantor A, Beil T, Whitlock EP. Screening for preeclampsia: a systematic evidence review for the U.S. preventive services task force. JAMA [Internet]. 2017 [acesso em 21 jan 2021]; 317(16):166883. Disponível em: https://www.ncbi.nlm.nih.gov/pubmed/28813 128Pregnancy

2. Medeiros AL, Santos SR, Cabral RWL, Silva JPG, Nascimento NM. Avaliando diagnósticos e intervenções de enfermagem no trabalho de parto e na gestação de risco. Rev Gaúcha Enferm [Internet]. 2016 [acesso em 21 jan. 2021];37(3):e55316. Disponível em: https://doi.org/10.1590/19831447.2016.03.55316

3. Fantasia HC. Low-dose aspirin for the prevention of preeclampsia. Nurs Womens Health [Internet]. 2018 [acesso em 21 jan 2021]; 22(1):87-92. Disponível em: https://doi.org/10.1016/j.nwh.2017.12.002

4. Rocha RS, Alves JAG, Moura SBMH, Araújo Júnior E, Peixoto AB, Santana EFM, et al. Simple approach based on maternal characteristics and mean arterial pressure for the prediction of preeclampsia in the first trimester of pregnancy. J Perinat Med [Internet]. 2017 [acesso em 21 jan 2021]; 
45(7):8439.

Disponível

em:
9 O'Gorman N, Wright D, Poon LC, Rolnik DL, Syngelaki A, Alvarado $M$, et al. Multicenter screening for pre-eclampsia by maternal factors and biomarkers at 11-13 weeks' gestation: comparison with NICE guidelines and ACOG recommendations. Ultrasound Obstet Gynecol [Internet]. 2017 [acesso em 22 jan 2021];49(06):756-760. Disponível em: 10.1002/ uog.17455

10. Oliveira AC, Santos AA, Bezerra AR, Barros AM, Tavares MC. Fatores Maternos e Resultados Perinatais Adversos em Portadoras de Pré-eclâmpsia em Maceió, Alagoas. Arq Bras. Cardiol [Internet]. 2016 [acesso em 22 jan 2021]; 106(2):113-20. Disponível em: 10.5935/abc.20150150.

e260. Disponível em: 10.1097/AOG.0000000000003891

7. O'Gorman N, Wright D, Syngelaki A, Akolekar R, Wright A, Poon LC, et al. Competing risks model in screening for preeclampsia by maternal factors and biomarkers at 11-13 weeks gestation. AJOG [Internet]. 2016 [acesso em 22 jan 2021]; 214(1): 103-e1. Disponível em: https://doi.org/10.1016/j.ajog.2015.08.034

8. Malachias MVB, Gomes MAM, Nobre F, Alessi A, Feitosa AD, Coelho EB. $7^{\mathrm{a}}$ Diretriz Brasileira de Hipertensão Arterial. Arq Bras Cardiol [Internet]. 2016 [acesso em 22 jan 2021]; 107(3):7-13. Disponível em: https://doi.org/10.5935/abc.20160152 https://doi.org/10.31011/reaid-2021-v.95-n.34-art.1069 Rev Enferm Atual In Derme v. 95, n. 34, 2021 e-021083
11. Ferreira ETM, Moura NS, Gomes MLS, Silva EG, Guerreiro MGS, Oriá MOB. Características maternas e fatores de risco para pré-eclâmpsia em gestantes. Rev Rene [Internet]. 2019 [acesso em 23 jan 2021]; 20:e40327. Disponível em: https://doi.org/10.15253/2175-

6783.20192040327

12. Amorim FCM, Neves ACN, Moreira FS, Oliveira ADS, Nery IS. Perfil de gestantes com pré-eclâmpsia. Rev Enferm UFPE online [Internet]. 2017 [acesso em 23 jan 2021]; 11(4):1574-83. Disponível em: 10.5205/reuol.9763-85423-1-SM.1104201703 
13. Gasparin VA, Albrecht CC, Favero DC, Gregolin KR, Pitilin EDB, Silva DTDR. Atividade física em gestantes como prevenção da síndrome hipertensiva gestacional. Rev Enferm UFPE on line [Internet]. 2018 [acesso em 23 jan 2021]; 12(4):1017-26. Disponível em: 10.5205/1981-8963-v12i4a230733p10171026-2018

14. Federação Brasileira das Associações de Ginecologia e Obstetrícia. Guia Prático: Infecções no Ciclo Gravídico Puerperal. Série orientações e recomendações. [Internet]. 2016 [acesso em 23 jan. 2021] Disponível em:https://www.febrasgo.org.br/media/k2/atta chments/02-

INFECCOyES_NO_CICLO_GRAVIDO_PU ERPERAL.pdf

15. Jacob L, Taskan S, Macharey G, Sechet I, Ziller V, Kostev K. Impact of caesarean section on mode of delivery, pregnancyinduced and pregancy-associated disorders, and complications in the subsequent pregnancy in Germany. Ger Medical Sci [Internet]. 2016 [acesso em 23 jan 2021]; 14(14):doc06. Disponível em: https://doi.org/10.3205/000233

16. Antunes MB, Demitto MO, Gravena AAF, Padovani C, Pelloso SM. Síndrome hipertensiva e resultados perinatais em gestação de alto risco . Rev Min Enferm[Internet]. 2017 [acesso em 23 jan
2021]; 21:e-1057. Disponível em: https://doi.org/10.5935/1415-2762.20170067

17. Burton GJ, Redman CW, Roberts JM, Moffett A. Pre-eclampsia: pathophysiology and clinical implications. BMJ [Internet]. 2019 [acesso em 23 jan 2021]; 366:12381. Disponível em: https://doi.org/10.1136/bmj.12381

Submissão: 2021-03-29

Aprovado: 2021-05-19 